\title{
Ring chromosome 9 syndrome
}

INSERM

\section{Source}

INSERM. (1999). Orphanet: an online rare disease and orphan drug data base. Ring chromosome 9 syndrome. ORPHA:96173

Ring chromosome 9 syndrome is an autosomal anomaly characterized by variable clinical features, most commonly including developmental delay, some degree of intellectual disability, facial dysmorphism, microcephaly, cong enital heart anomalies, and variable genital, limb and skeletal anomalies. 